\title{
Follow-up of GWTC-2 gravitational wave events with neutrinos from the Super-Kamiokande detector
}

\section{Mathieu Lamoureux ${ }^{a, *}$ on behalf of the Super-Kamiokande Collaboration}

(a complete list of authors can be found at the end of the proceedings)

${ }^{a}$ Dipartimento di Fisica, INFN Sezione di Padova and Università di Padova, I-35131, Padova, Italy

E-mail: mathieu.lamoureux@pd.infn.it

Super-Kamiokande (SK) is a 50-kt water Cherenkov detector, instrumented with $\sim 13 \mathrm{k}$ photomultipliers and running since 1996. It is sensitive to neutrinos with energies ranging from $4.5 \mathrm{MeV}$ to several TeV. A new framework has been developed for the follow-up of gravitational wave (GW) alerts issued by the LIGO-Virgo collaboration (LVC). Neutrinos are searched for, using a 1000second time window centered on the alert time and in both SK low-energy and high-energy samples. Such observation can then be used to constrain the neutrino emission from the GW source. The significance of potential signals has been obtained by comparing neutrino direction with the localization of the GW. The computation of limits on incoming neutrino flux and on the total energy emitted in neutrinos by the source has been performed for the different neutrino flavors. The results using the LVC GWTC-2 catalog (covering O3a period) are presented, as well as the outlooks for the future real-time public release of follow-ups for the $\mathrm{O} 4$ period (in 2022) and beyond.

$37^{\text {th }}$ International Cosmic Ray Conference (ICRC 2021)

July 12th - 23rd, 2021

Online - Berlin, Germany

\footnotetext{
${ }^{*}$ Presenter
} 


\section{Introduction}

The LIGO/Virgo collaboration (LVC) has released GWTC-2, their new catalog of gravitational waves (GW) objects, detected during the first half of the third observing run (O3a) from April to September 2019 [1]. The detection of neutrinos in time and spatial coincidence with any of these objects would be the first hint for a joint source emitting both messengers and it would greatly help to understand the underlying mechanisms.

Super-Kamiokande [2] is a Cherenkov detector located in the Mozumi mine in Japan. It consists in a tank of $50 \mathrm{kt}$ of water, separated into an inner detector ( $32 \mathrm{kt}$ ) equipped with $\sim 11,000$ PMTs and an outer detector used mainly as veto. The data can be broadly divided into four sub-samples:

- low-energy neutrinos (LE- $\left.v, 7 \mathrm{MeV}<E_{v}<100 \mathrm{MeV}\right)$,

- high-energy fully-contained events (HE- $\left.v / \mathrm{FC}, 0.1 \mathrm{GeV}<E_{v} \gtrsim 10 \mathrm{GeV}\right)$,

- high-energy partially-contained events(HE- $\left.v / \mathrm{PC}, 0.1 \mathrm{GeV}<E_{v} \gtrsim 100 \mathrm{GeV}\right)$,

- and high-energy upgoing muons (HE- $v / \mathrm{UPMU}, E_{v} \sim O(\mathrm{GeV}-\mathrm{TeV})$ ).

The former is mainly sensitive to electron anti-neutrinos from inverse beta decay and other flavors contribute through elastic scatterings. The FC and PC samples are sensitive to $v_{\mu}, \bar{v}_{\mu}, v_{e}$, and $\bar{v}_{e}$, while the UPMU sample is focusing on muon (anti-)neutrinos originating below the SK horizon (downgoing events are not selected due to large atmospheric muon background).

The data from the detector have been used to search for neutrinos in time coincidence with GW objects, using a $1000 \mathrm{~s}$ time window centered on each trigger. For the three high-energy samples, the spatial correlation between the reconstructed neutrino direction and the GW localization is used to assign p-values to the observations and upper limits on the emitted neutrino flux have been computed. Detailed methods and results are presented in [3] and the data release [4] contains the full list of observations and flux limits for the $39 \mathrm{GW}$ in the GWTC-2 catalog.

The section 2 presents an overview of the event-by-event results of the $39 \mathrm{GW}$ follow-ups while the section 3 focuses on the joint analysis of binary mergers in order to constrain their population. Lastly, the section 4 highlights the important results and the prospects for future observations.

\section{SK observations}

Neutrinos have been searched for in $1000 \mathrm{~s}$ time window centered on each GW events from the GWTC-2 catalog. Out of the $39 \mathrm{GWs}$, the follow-up was possible for 36 of them (35 for low-energy energy neutrinos), as the detector data are not available for 3 of them, due to calibration periods. The number of observed events is compared to the expectation that is based on the average background rate for each sample and on the detector livetime over the time window. The expected backgrounds from solar and atmospheric neutrinos in $1000 \mathrm{~s}$ are $0.729,0.129,0.007$, and 0.015 for LE- $v$, HE- $v /$ FC, HE- $v /$ PC, and HE- $v /$ UPMU samples, respectively.

The Figure 1 summarizes the follow-up of all events. No significant excess was observed in any of the four samples: $N_{\mathrm{obs}}=24,8,0,2$ observed events respectively with the same order as above, compared to $N_{\exp }=25.0,4.0,0.3,0.6$ expected background events summing over the full list. 


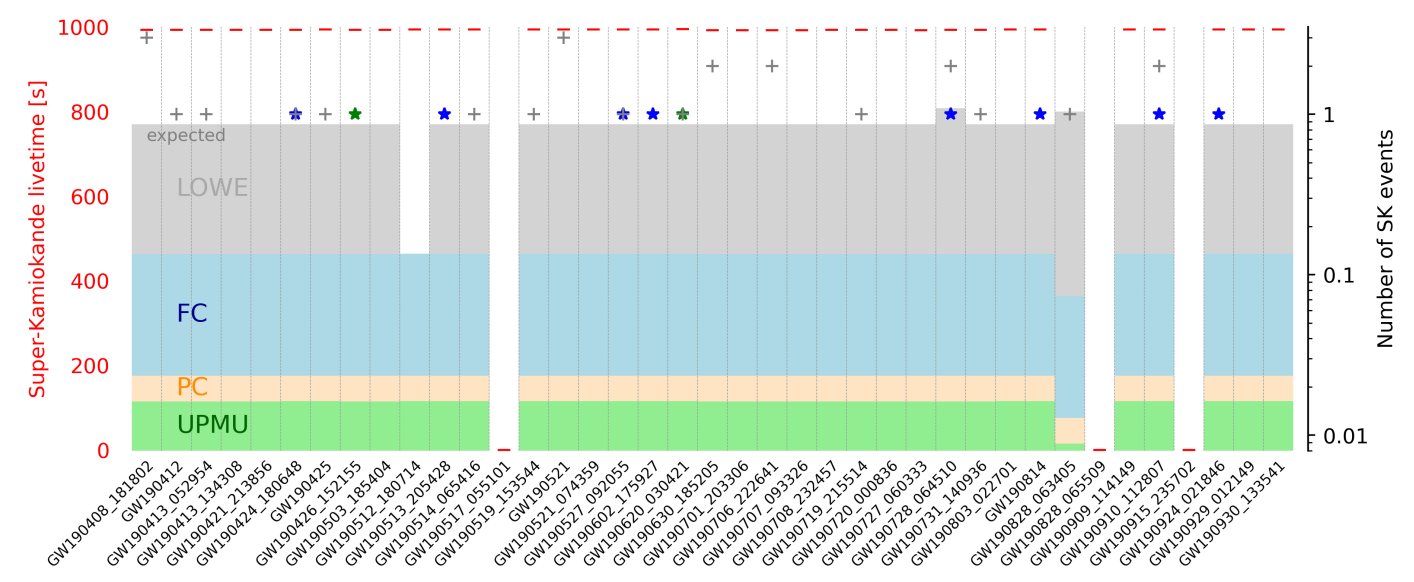

Figure 1: Summary of the follow-up of GWTC-2 events. The red plot shows the Super-Kamiokande livetime over the selected $1000 \mathrm{~s}$ window for each trigger. The light color stacked bars indicate the expected number of events and the markers give the observed number of events for the four samples.

For the ten observed events in the HE- $v / \mathrm{FC}$ and HE- $v /$ UPMU samples, it is possible to use the reconstructed neutrino direction in order to better quantify the "signalness" of the observations (the skymaps are available e.g. in [4]). The method, detailed in [3], consists in defining a test statistic $T S$ to discriminate between background events (covering the full sky and following atmospheric neutrino spectrum) and signal events (point source consistent with GW localization, $E^{-\gamma}$ spectrum).

The final p-value can be dissociated into $p=p_{\text {time }} \times p_{\text {space }}$ where $p_{\text {time }}$ is the Poisson p-value associated to the observation of at least one event given the expected background (for $N_{\exp }=$ $N_{\text {exp }, \text { FC }}+N_{\text {exp,PC }}+N_{\text {exp, UPMU }}=0.13$, we get $\left.p_{\text {time }}=12.6 \%\right)$ and $p_{\text {space }}$ is obtained comparing observed value of $T S$ to the expected background distribution obtained from MC simulations (with at least one neutrino event).

The Figure 2 illustrates the obtained p-values. The most significant observation is associated to GW190602_175927 with a pre-trial p-value of $0.22 \%$. However, it is needed to take into account the corresponding trial factor (as the analysis was repeated 36 times, for the 36 follow-ups) and the final post-trial p-value is $7.8 \%$ (equivalent to $1.4 \sigma$ ), hence being fully compatible with the background-only hypothesis.

Upper limits on the incoming neutrino flux have been computed for each GW and full results are available in [4]. For the LE- $v$ sample, limits were obtained with the assumption of a FermiDirac spectrum and typical limits are of the order of $10^{8} \mathrm{~cm}^{-2}$ for the $\bar{v}_{e}$ fluence at $90 \%$ C.L. $\left(10^{9}-10^{10} \mathrm{~cm}^{-2}\right.$ for other flavors). For the three HE- $v$ samples, limits were computed assuming $E^{-2}$ spectrum, first independently for each sample but also by combining them. As the UPMU sample is only sensitive to events below the horizon, high-energy limits feature two different behaviors: for GW mainly located below the horizon, the UPMU sample is effectively used and we get $E^{2} \frac{\mathrm{d} n}{\mathrm{~d} E}<40 \mathrm{GeV} \mathrm{cm}^{-2}$ (90\% C.L.); otherwise, only FC and PC are contributing and $E^{2} \frac{\mathrm{d} n}{\mathrm{~d} E}<2 \times 10^{3} \mathrm{GeV} \mathrm{cm}^{-2}$.

Detailed results for low-energy and high-energy samples are presented in [4]. 


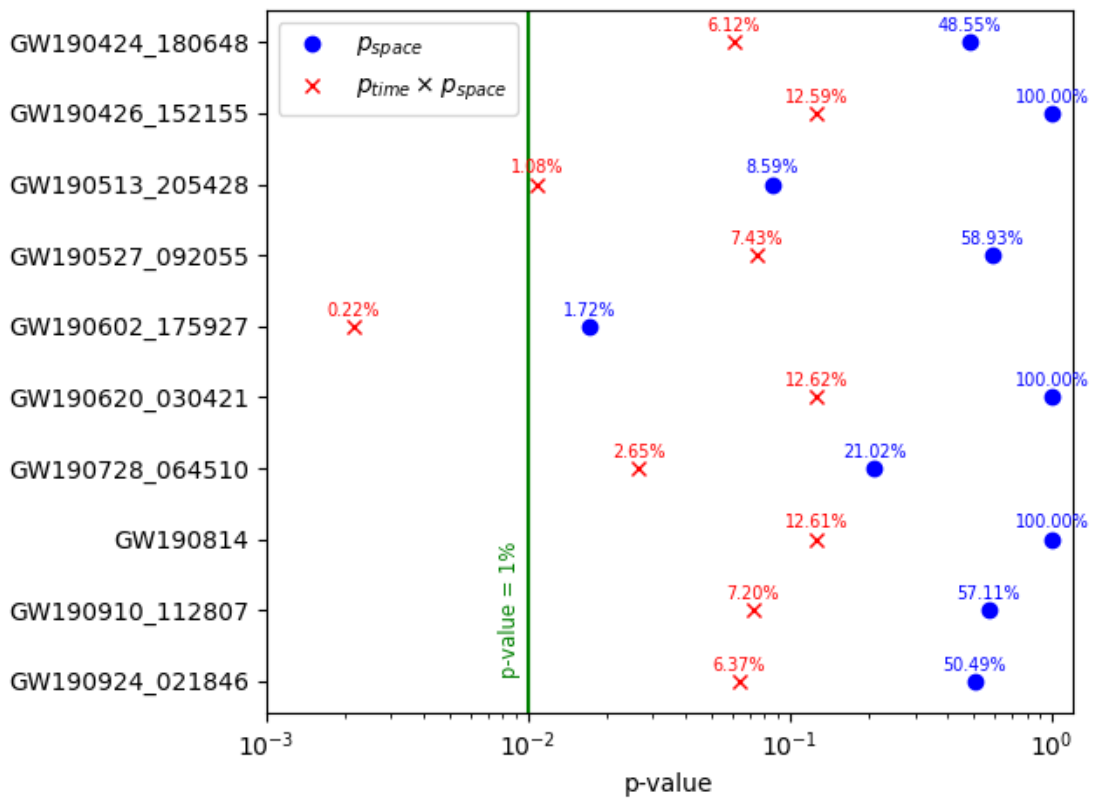

Figure 2: Obtained p-values for the ten observed events in the HE- $v$ samples. The $p_{\text {space }}$ values are reported with blue markers while the total p-values are shown in red.

\section{Population constraints with high-energy samples}

As for the neutrino flux, upper limits on the total energy $E_{\text {iso }}$ emitted in neutrinos by the source (assuming isotropic emission) can be obtained by taking into account the source luminosity distance $d$. However, its correlation with the localization has to be considered by using the 3D skymaps (in right ascension, declination and distance) from the GWTC-2 catalog. The full method is described in [3].

The Figure 3 shows the obtained limits on all-flavor $E_{\text {iso }}$ for $E^{-2}$ spectrum assuming the flux at Earth is equally distributed between the neutrino flavors $\left(v_{e} / \bar{v}_{e}, v_{\mu} / \bar{v}_{\mu}, v_{\tau} / \bar{v}_{\tau}\right.$, where the contribution of the latter in SK samples is neglected in this analysis). This hypothesis of equipartition originates from the most common source scenario where the produced flavor ratio is $v_{e}: v_{\mu}: v_{\tau}=1: 2: 0$ which becomes $1: 1: 1$ after oscillations on astronomical distance. The two-tiers behavior introduced in the last section is clearly visible, with the limits being distributed along two lines depending whether the UPMU contribution is significant or not.

The list of GWTC-2 objects followed by SK is populated with 33 binary black hole mergers (BBH), making it a very good sample to constrain the neutrino emission from these objects. Individual upper limits presented in Figure 3 can thus be combined under the assumption that either (a) all $\mathrm{BBH}$ emits the same total energy $E_{\text {iso }}$ in neutrinos or (b) $E_{\text {iso }}$ is proportional to the total mass of the binary system: $E_{\text {iso }}=f_{v} \times \mathcal{M}_{\text {tot }}$ (where $\mathcal{M}_{\text {tot }}$ is obtained from the GWTC-2 catalog). These two hypotheses were investigated and the following limits were obtained:

$$
\begin{aligned}
& \text { (a) } E_{\text {iso }}^{\text {per } \mathrm{BBH}}<4.16 \times 10^{55} \mathrm{erg} \\
& \text { (b) } f_{v}<1.06 \times 10^{54} \mathrm{erg} / \mathrm{M}_{\odot}=66 \%
\end{aligned}
$$




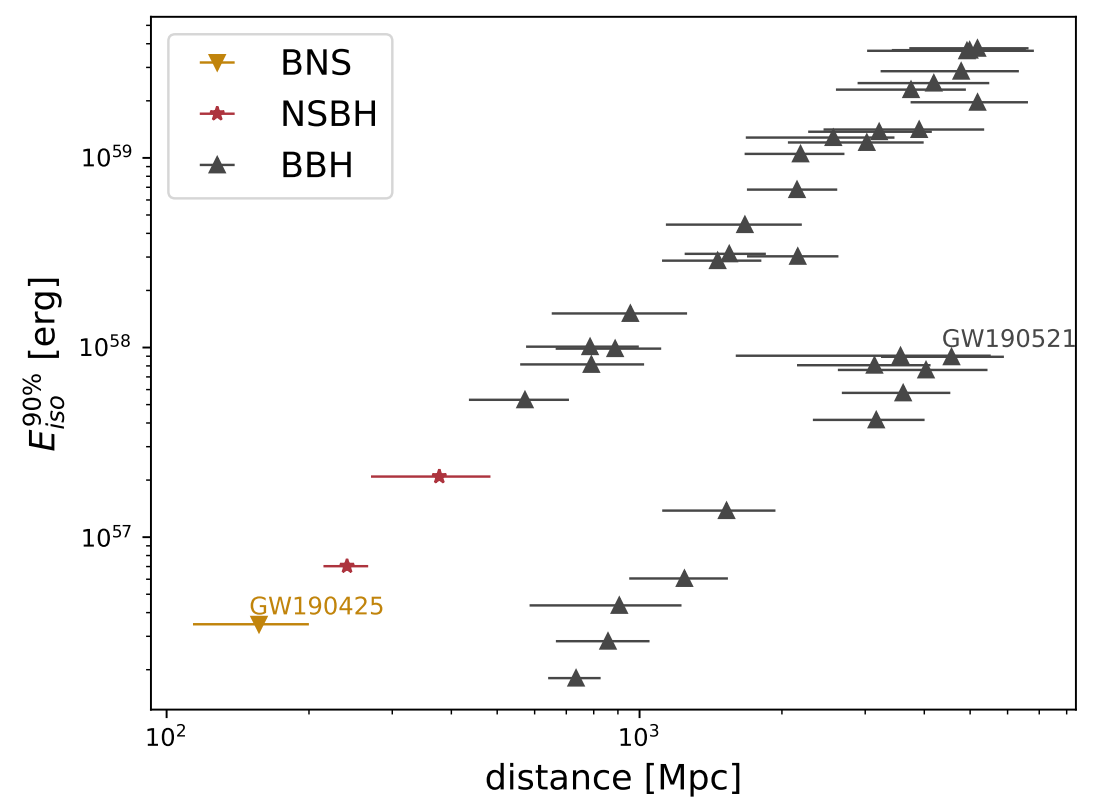

Figure 3: Upper limits on $E_{\text {iso }}$ for the $36 \mathrm{GW}$ triggers followed up by SK, as a function of source mean distance, assuming equipartition between all neutrino flavors.

\section{Discussion and conclusion}

The results presented in the previous sections yield no significant observations and upper limits have been computed. The final results are the $90 \%$ upper limits on $E_{\text {iso }}$ for each trigger and from the joint study for BBH objects. One can notice that the obtained limits for $E^{-2}$ spectrum are barely covering the unrealistic region where most than half of the initial mass of the binary system is converted to neutrinos, but the situation may greatly change if the assumed spectrum is different (e.g. $E^{-2}$ spectrum with a cut-off or softer spectrum).

Moreover, the GWTC-2 catalog only contains three mergers where neutron stars are likely involved and all these events were located above the SK horizon. The follow-up of mergers during the next GW observation periods are very promising, in particular in the case of binary neutron star mergers (where large neutrino emission is expected [5]) below the SK horizon (where the UPMU sample is expected to contribute). In this context, the possibility to perform real-time follow-up of public alerts with Super-Kamiokande may contribute to the discovery of a joint $\mathrm{GW}+v$ source, that would complete the triangle of real-time multimessenger observations between neutrinos, electromagnetic and gravitational waves (TXS 0506+056 being the foremost example of coincidence between the two former, and GW170817 for the two latter).

\section{Acknowledgments}

The work of the presenter has received funding from the European Union's Horizon 2020 research and innovation program under the Marie Skłodowska-Curie grant agreement No 754496. The full author list and acknowledgments of the collaboration are presented in [3]. 


\section{References}

[1] R. Abbott et al. [LIGO Scientific and Virgo], [arXiv:2010.14527 [gr-qc]].

[2] Y. Fukuda et al. [Super-Kamiokande], Nucl. Instrum. Meth. A 501 (2003), 418-462

[3] K. Abe et al. [Super-Kamiokande], [arXiv:2104.09196 [astro-ph.HE]].

[4] K. Abe et al. [Super-Kamiokande], doi:10.5281/zenodo.4724822

[5] S. S. Kimura et al., Phys. Rev. D 98 (2018) no.4, 043020 


\section{Full Authors List: Super-Kamiokande Collaboration}

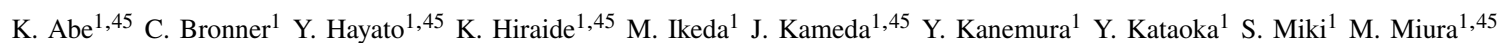
S. Moriyama ${ }^{1,45}$ Y. Nagao ${ }^{1}$ M. Nakahata ${ }^{1,45}$ S. Nakayama ${ }^{1,45}$ K. Okamoto $^{1}$ G. Pronost ${ }^{1}$ H. Sekiya ${ }^{1,45}$ M. Shiozawa ${ }^{1,45}$ Y. Sonoda $^{1}$ Y. Suzuki ${ }^{1}$ A. Takeda ${ }^{1,45}$ Y. Takemoto ${ }^{1}$ A. Takenaka ${ }^{1}$ H. Tanaka ${ }^{1}$ S. Watanabe ${ }^{1}$ T. Yano ${ }^{1}$ S. Han ${ }^{2}$ T. Kajita ${ }^{2,45}$ K. Okumura ${ }^{2,45}$ T. Tashiro ${ }^{2}$ J. Xia ${ }^{2}$ G. D. Megias ${ }^{3}$ L. Labarga ${ }^{4}$ Ll. Marti ${ }^{4}$ B. Zaldivar ${ }^{4}$ B. W. Pointon ${ }^{6,49}$ E. Kearns ${ }^{5,45}$ J. L. Raaf ${ }^{5}$ L. Wan ${ }^{5}$ T. Wester ${ }^{5}$

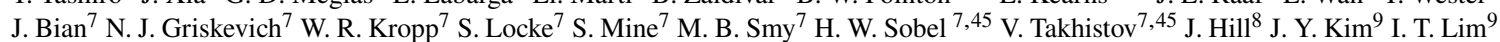
R. G. Park ${ }^{9}$ B. Bodur ${ }^{10}$ K. Scholberg ${ }^{10}$ C. W. Walter ${ }^{10,45}$ L. Bernard ${ }^{11}$ A. Coffani ${ }^{11}$ O. Drapier ${ }^{11}$ S. El Hedri ${ }^{11}$ A. Giampaolo ${ }^{11}$ M. Gonin ${ }^{11}$ Th. A. Mueller ${ }^{11}$ P. Paganini ${ }^{11}$ B. Quilain ${ }^{11}$ T. Ishizuka ${ }^{12}$ T. Nakamura ${ }^{13}$ J. S. Jang ${ }^{14}$ J. G. Learned ${ }^{15} \mathrm{~S} \mathrm{Cao}^{16}$ L. H. V. Anthony ${ }^{17}$ D. Martin ${ }^{17}$ M. Scott ${ }^{17}$ A. A. Sztuc ${ }^{17}$ Y. Uchida ${ }^{17}$ V. Berardi ${ }^{18}$ M. G. Catanesi ${ }^{18}$ E. Radicioni ${ }^{18}$ N. F. Calabria ${ }^{19}$

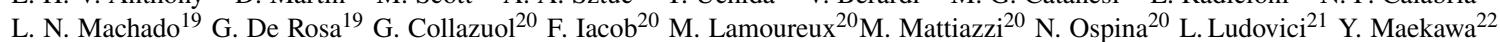
Y. Nishimura ${ }^{22}$ M. Friend ${ }^{23}$ T. Hasegawa ${ }^{23}$ T. Ishida ${ }^{23}$ T. Kobayashi ${ }^{23}$ M. Jakkapu ${ }^{23}$ T. Matsubara ${ }^{23}$ T. Nakadaira ${ }^{23}$ K. Nakamura ${ }^{23,45}$ Y. Oyama ${ }^{23}$ K. Sakashita ${ }^{23}$ T. Sekiguchi ${ }^{23}$ T. Tsukamoto ${ }^{23}$ T. Boschi ${ }^{24}$ F. Di Lodovico ${ }^{24}$ J. Gao ${ }^{24}$ J. Migenda ${ }^{24}$ M. Taani ${ }^{24}$ S. Zsoldos ${ }^{24}$ Y. Nakano ${ }^{25}$ H. Ozaki $^{25}$ A. T. Suzuki ${ }^{25}$ Y. Takeuchi ${ }^{25,45}$ S. Yamamoto ${ }^{25}$ Y. Kotsar ${ }^{25}$ A. Ali ${ }^{26}$ J. Feng ${ }^{26}$ T. Kikawa ${ }^{26}$ M. Mori $^{26}$ T. Nakaya ${ }^{26,45}$ R. A. Wendell ${ }^{26,45}$ K. Yasutome ${ }^{26}$ P. Fernandez ${ }^{28}$ N. McCauley ${ }^{28}$ P. Mehta $^{28}$ K. M. Tsui ${ }^{27}$ Y. Fukuda ${ }^{28}$ Y. Itow ${ }^{29,30}$ H. Menjo ${ }^{29}$ K. Sato ${ }^{29}$ J. Lagoda ${ }^{31}$ S. M. Lakshmi ${ }^{31}$ P. Mijakowski ${ }^{31}$ J. Zalipska ${ }^{31}$ J. Jiang ${ }^{32}$ C. K. Jung ${ }^{32}$ C. Vilela ${ }^{32}$ M. J. Wilking ${ }^{32}$

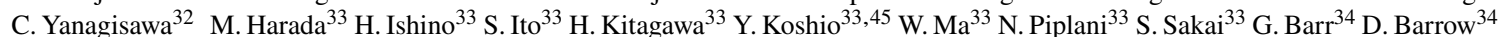
L. Cook ${ }^{34,45}$ A. Goldsack ${ }^{34,45}$ S. Samani ${ }^{34}$ D. Wark ${ }^{34,39}$ F. Nova ${ }^{35}$ J. Y. Yang ${ }^{36}$ S. J. Jenkins ${ }^{37}$ M. Malek ${ }^{37}$ J. M. McElwee ${ }^{37}$ O. Stone ${ }^{37}$

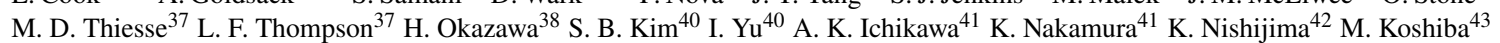
K. Iwamoto ${ }^{44}$ Y. Nakajima ${ }^{44}$ N. Ogawa ${ }^{44}$ M. Yokoyama $a^{44,45}$ K. Martens ${ }^{45}$ M. R. Vagins ${ }^{45,7}$ M. Kuze ${ }^{46}$ S. Izumiyama ${ }^{46}$ M. Inomoto ${ }^{47}$ M. Ishitsuka ${ }^{47}$ H. Ito ${ }^{47}$ T. Kinoshita ${ }^{47}$ R. Matsumoto ${ }^{47}$ M. Shinoki ${ }^{47}$ T. Suganuma ${ }^{47}$ J. F. Martin ${ }^{48}$ H. A. Tanaka ${ }^{48}$ T. Towstego ${ }^{48}$ R. Akutsu ${ }^{49}$ P. de Perio ${ }^{49}$ M. Hartz ${ }^{49}$ A. Konaka ${ }^{49}$ N. W. Prouse ${ }^{49}$ S. Chen $^{50}$ B. D. Xu ${ }^{50}$ M. Posiadala-Zezula ${ }^{51}$ D. Hadley ${ }^{52}$

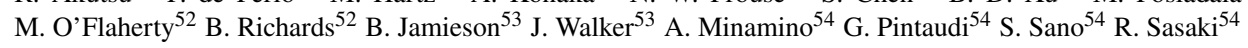

${ }^{1}$ Kamioka Observatory, Institute for Cosmic Ray Research, University of Tokyo, Kamioka, Gifu 506-1205, Japan.

${ }^{2}$ Research Center for Cosmic Neutrinos, Institute for Cosmic Ray Research, University of Tokyo, Kashiwa, Chiba 277-8582, Japan.
2 , Institute for Cosmic Ray Resarch, University of Tokyo, Kamika,

${ }^{3}$ Institute for Cosmic Ray Research, University of Tokyo, Kashiwa, Chiba 277-8582, Japan.

${ }^{3}$ Institute for Cosmic Ray Research, University of Tokyo, Kashiwa, Chiba 277-8582, Japan.
${ }^{4}$ Department of Theoretical Physics, University Autonoma Madrid, 28049 Madrid, Spain.

${ }^{4}$ Department of Theoretical Physics, University Autonoma Madrid, 28049
${ }^{5}$ Department of Physics, Boston University, Boston, MA 02215, USA.

${ }^{6}$ Department of Physics, Boston University, Boston, MA 02215, USA.

${ }^{7}$ Department of Physics, British Columbia Institute of Technology, Burnaby, BC, V5G 3H2, Canada.

${ }^{8}$ Department of Physics and Astronomy, University of California, Irvine, Irvine, CA 92697-4575,
${ }^{7}$ Department of Physics, California State University, Dominguez Hills, Carson, CA 90747, USA.

${ }^{8}$ Department of Physics, California State University, Dominguez Hills, Carson, CA 90747, USA.
${ }^{9}$ Institute for Universe and Elementary Particles, Chonnam National University, Gwangju 61186, Korea.

${ }^{9}$ Institute for Universe and Elementary Particles, Chonnam National U.
${ }^{10}$ Department of Physics, Duke University, Durham NC 27708, USA.

${ }^{11}$ Ecole Polytechnique, IN2P3-CNRS, Laboratoire Leprince-Ringuet, F-91120 Palaiseau, France.

${ }^{12}$ Junior College, Fukuoka Institute of Technology, Fukuoka, Fukuoka 811-0295, Japan.

${ }^{3}$ Department of Physics, Gifu University, Gifu, Gifu 501-1193, Japan.

${ }^{14}$ GIST College, Gwangju Institute of Science and Technology, Gwangju 500-712, Korea.

${ }^{5}$ Department of Physics and Astronomy, University of Hawaii, Honolulu, H 96822 , USA.

${ }^{16}$ Institute For Interdisciplinary Research in Science and Education, ICISE, Quy Nhon, 55121, Vietnam.

${ }^{17}$ Department of Physics, Imperial College London, London, SW7 2AZ, United Kingdom

${ }^{18}$ Dipartimento Interuniversitario di Fisica, INFN Sezione di Bari and Università e Politecnico di Bari, I-70125, Bari, Italy.

${ }^{19}$ Dipartimento di Fisica, INFN Sezione di Napoli and Università di Napoli, I-80126, Napoli, Italy.

${ }^{20}$ Dipartimento di Fisica, INFN Sezione di Padova and Università di Padova, I-35131, Padova, Italy.

${ }^{21}$ INFN Sezione di Roma and Università di Roma "La Sapienza", I-00185, Roma, Italy.

22 Department of Physics, Keio University, Yokohama, Kanagawa, 223-8522, Japan.

${ }^{23}$ High Energy Accelerator Research Organization (KEK), Tsukuba, Ibaraki 305-0801, Japan .

25epartment of Physics, King's College London, London, WC2R 2LS, UK

${ }^{26}$ Department of Physics, Kobe University, Kobe, Hyogo 657-8501, Japan

${ }^{27}$ Department of Physics, Kyoto University, Kyoto, Kyoto 606-8502, Japan.

${ }^{27}$ Department of Physics, University of Liverpool, Liverpool, L69 7ZE, United Kingdom.

${ }^{29}$ Institute for Space-Earth Environmental Research, Nagoya University, Nagoya, Aichi 464-8602, Japan.

${ }^{30}$ Kobayashi-Maskawa Institute for the Origin of Particles and the Universe, Nagoya University, Nagoya, Aichi 464-8602, Japan.

${ }^{31}$ National Centre For Nuclear Research, 02-093 Warsaw, Poland.

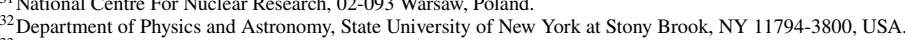

${ }^{33}$ Department of Physics and Astronomy, State University of New York at Stony Brook,
${ }^{3}$ Department of Physics, Okayama University, Okayama, Okayama 700-8530, Japan

${ }^{34}$ Department of Physics, Oxford University, Oxford, OX1 3PU, United Kingdom.

${ }^{34}$ Department of Physics, Oxford University, Oxford, OX1 3PU, United
${ }^{3}$ Rutherford Appleton Laboratory, Harwell, Oxford, OX11 0QX, UK.

${ }^{36}$ Department of Physics, Seoul National University, Seoul 151-742, Korea.

${ }^{37}$ Department of Physics and Astronomy, University of Sheffield, S3 7RH, Sheffield, United Kingdom.

${ }^{38}$ Department of Physics and Astronomy, University of Sheffield, S3 7RH, Sheffield, United Kingdom.

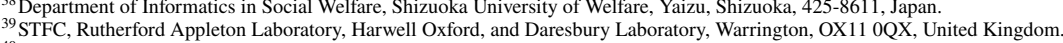

${ }^{39}$ STFC, Rutherford Appleton Laboratory, Harwell Oxford, and Daresbury La
${ }^{40}$ Department of Physics, Sungkyunkwan University, Suwon 440-746, Korea.

${ }^{41}$ Department of Physics, Faculty of Science, Tohoku University, Sendai, Miyagi, 980-8578, Japan.

${ }^{42}$ Department of Physics, Tokai University, Hiratsuka, Kanagawa 259-1292, Japan.

${ }^{43}$ The University of Tokyo, Bunkyo, Tokyo 113-0033, Japan.

${ }^{4}$ Department of Physics, University of Tokyo, Bunkyo, Tokyo 113-0033, Japan.

${ }^{4}$ Kavli Institute for the Physics and Mathematics of the Universe (WPI), The University of Tokyo Institutes for Advanced Study, University of Tokyo, Kashiwa, Chiba 277-8583, Japan.

${ }^{46}$ Department of Physics, Tokyo Institute of Technology, Meguro, Tokyo 152-8551, Japan.

${ }^{47}$ Department of Physics, Faculty of Science and Technology, Tokyo University of Science, Noda, Chiba 278-8510, Japan.

${ }^{48}$ Department of Physics, University of Toronto, ON, M5S 1A7, Canada.

${ }^{9}$ TRIUMF, 4004 Wesbrook Mall, Vancouver, BC, V6T2A3, Canad

${ }^{0}$ Department of Engineering Physics, Tsinghua University, Beijing, 100084, China.

${ }^{51}$ Faculty of Physics, University of Warsaw, Warsaw, 02-093, Poland.

53 Department of Physics, University of Warwick, Coventry, CV4 7AL, UK.

${ }^{54}$ Department of Physics, Yokohama National University, Yokohama, Kanagawa, 240-8501, Japan.

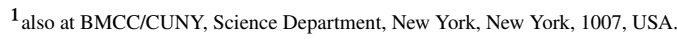

${ }^{2}$ Deceased. 